\title{
Prophylactic peritoneal dialysis catheter does not decrease time to achieve a negative fluid balance after the Norwood procedure: A randomized controlled trial
}

\author{
Lindsay M. Ryerson, MD, ${ }^{a}$ Andrew S. Mackie, MD, SM, ${ }^{\mathrm{a}}$ Joseph Atallah, MD, SM, ${ }^{\mathrm{b}}$ Ari R. Joffe, MD, ${ }^{\mathrm{a}}$ \\ Ivan M. Rebeyka, MD, ${ }^{\mathrm{b}}$ David B. Ross, MD, ${ }^{\mathrm{b}}$ and Ian Adatia, $\mathrm{MBChB}^{\mathrm{a}}$
}

\begin{abstract}
Objective: Infants and children who undergo cardiopulmonary bypass and cardiac surgery are at risk of postoperative fluid overload. Peritoneal dialysis catheter (PDC) and peritoneal dialysis are reported to be effective means of postoperative fluid management. We sought to test the hypothesis that PDC insertion in the operating room at the time of Norwood palliation would decrease the time to achieve a negative fluid balance in a group of neonates with hypoplastic left heart syndrome.
\end{abstract}

\begin{abstract}
Methods: A single center randomized controlled trial was performed. We randomized neonates with hypoplastic left heart syndrome to prophylactic PDC, with or without dialysis, or standard care (ie, no PDC).

Results: Twenty-two neonates were included; 10 were randomized to PDC and 12 were randomized to standard care. The mean time to first postoperative negative fluid balance was $2.70 \pm 1.06$ days for the prophylactic PDC group and $2.67 \pm 0.65$ days for the standard care group $(P=.93)$. There was no difference between the 2 groups in time to lactate $\leq 2 \mathrm{mmol} / \mathrm{L}$, maximum vasoactive-inotrope score on postoperative days 2 to 5 , time to sternal closure, time to first extubation, modified clinical outcome score, or hospital length of stay. Twenty-one patients (95\%) survived to hospital discharge. Four patients randomized to prophylactic PDC had 1 or more serious adverse events compared with no patients in the standard care group $(P=.03)$.
\end{abstract}

Conclusions: Prophylactic PDC, with or without dialysis, did not decrease the time to achieve a negative fluid balance after the Norwood procedure, did not alter physiological variables postoperatively, and was associated with more severe adverse events. (J Thorac Cardiovasc Surg 2015;149:222-8)

See related commentary on pages 228-9.

Palliation for hypoplastic left heart syndrome (HLHS) with the Norwood procedure was first described in $1980^{1}$ and since then has undergone several modifications. Infants and children who undergo Norwood palliation and other complex cardiac surgical procedures are at risk for the development of acute kidney injury characterized by oliguria, hyperkalemia, and fluid retention with a reported incidence of $4 \%$ to $17 \%{ }^{2-6}$ and associated mortality of $18 \%$ to $57.5 \% .^{2-9}$ Even without a diagnosis of acute kidney injury, extravascular fluid retention is a common

From the Departments of Pediatrics ${ }^{\mathrm{a}}$ and Surgery, ${ }^{\mathrm{b}}$ University of Alberta, Edmonton, Alberta, Canada.

Supported by the Women and Children's Health Research Institute, Edmonton, Alberta, Canada.

Disclosures: Ian Adatia reports consulting fees for Eli Lilly and CDDI. All other authors have nothing to disclose with regard to commercial support.

Received for publication June 24, 2014; revisions received Aug 5, 2014; accepted for publication Aug 9, 2014; available ahead of print Sept 11, 2014.

Address for reprints: Lindsay M. Ryerson, MD, 3A3.19 WMC Stollery Children's Hospital, 8440 112th St, Edmonton, AB T6G 2B7, Canada (E-mail: ryerson@ ualberta.ca)

0022-5223/\$36.00

Copyright (C) 2015 by The American Association for Thoracic Surgery

http://dx.doi.org/10.1016/j.jtcvs.2014.08.011 problem after congenital cardiac surgery due to capillary leak. ${ }^{10}$ This may result in decreased preload as well as pulmonary and systemic tissue edema that may impair gas exchange. Elevated intra-abdominal pressure secondary to ascites may further compromise cardiac output ${ }^{11}$ and renal perfusion. ${ }^{12}$ Renal dysfunction exacerbates these ill effects by decreasing the clearance of water and solute, which delays reaching a negative fluid balance. Fluid overload is associated with poor outcomes in infants following congenital heart surgery. ${ }^{13}$

Multiple centers have retrospectively examined their experience with peritoneal dialysis (PD) in postoperative cardiac patients. PD increased urine output, ${ }^{6,8,14}$ achieved a net negative fluid balance, ${ }^{3,6,8,14}$ as well as decreased the mean number of inotropic agents required. ${ }^{7,8,14}$ The majority of patients reported to date have undergone cardiac surgery involving ventricular septal defect closure with insertion of right ventricle to pulmonary artery conduit; there is overrepresentation of patients at risk of developing restrictive right ventricular physiology postoperatively. However the postoperative physiology following the modified Norwood operation is distinctly different and the value of postoperative PD less clear.

Therefore we sought to test the hypothesis that peritoneal dialysis catheter (PDC) insertion in the operating room at the time of Norwood palliation will decrease the time to 

Abbreviations and Acronyms
$\mathrm{CPR}=$ cardiopulmonary resuscitation
ECLS = extracorporeal life support
HLHS = hypoplastic left heart syndrome
LCOS = low cardiac output syndrome
LOS = length of stay
PCICU $=$ pediatric cardiac intensive care unit
PDC = peritoneal dialysis catheter
$\mathrm{Q}_{\mathrm{s}} \quad=$ systemic blood flow
SVRi $=$ indexed systemic vascular resistance
VIS $=$ vasoactive-inotrope score

achieve a negative fluid balance in a group of neonates with HLHS and its variants. Secondary aims were to assess the influence of PDC on systemic blood flow $\left(\mathrm{Q}_{\mathrm{s}}\right)$, indexed systemic vascular resistance (SVRi), time to lactate $\leq 2 \mathrm{mmol} / \mathrm{L}$, maximum vasoactive-inotrope score (VIS) ${ }^{15}$ on postoperative days 2 to 5 , time to sternal closure, time to first extubation, modified clinical outcome score, ${ }^{16}$ and hospital length of stay (LOS).

\section{METHODS \\ Study Design}

This randomized controlled trial was conducted in the pediatric cardiac intensive care unit (PCICU) at the Stollery Children's Hospital in Edmonton, Canada. Neonates with HLHS or its variants scheduled to undergo a Norwood procedure were eligible. Exclusion criteria included premature neonates $<37$ weeks gestation and weight $<2.0 \mathrm{~kg}$ because these patients are at higher risk of poor outcomes after Norwood procedure, urine output $<0.5 \mathrm{~mL} / \mathrm{kg} / \mathrm{h}$ over 24 hours in the 48 hours before the Norwood procedure, any preoperative renal replacement therapy within 48 hours of the Norwood procedure, abdominal defects precluding placement of a PDC, documented chromosomal abnormality, preoperative cardiopulmonary resuscitation (CPR), or preoperative extracorporeal life support (ECLS). Our institutional health ethics review board approved the study. Written and informed consent was obtained from parents or legal guardians. The trial was registered with clinicaltrials.gov (identifier no. NCT01215240).

\section{Interventions and Outcomes}

Study participants were randomly assigned to receive a PDC placed in the operating room at the time of the Norwood procedure or standard care (ie, no prophylactic PDC). For those patients randomized to prophylactic PDC, a cuffed PDC (Tenchkoff catheter) was placed by the direct transperitoneal method by the cardiac surgeon (I.M.R. or D.B.R.). ${ }^{17}$ Randomization occurred by central concealed Web-based block randomization in groups of 4 and stratified by surgeon. Standard cardiopulmonary bypass techniques were employed in all patients maintaining hemoglobin levels $>10 \mathrm{~g} / \mathrm{dL}$ with maximum flow rates of $150 \mathrm{~mL} / \mathrm{kg} / \mathrm{min}$. The Norwood procedure was performed with a right-ventricle to pulmonary artery conduit in all participants, and sternal closure was routinely delayed. Modified ultrafiltration was performed in the operating room for all participants. The study protocol was initiated immediately upon postoperative admission to the PCICU. All clinicians were aware of the study group assignment because it was not possible to blind placement of the PDC.

Participants randomized to prophylactic PDC had their PDC left open to free drainage in the PCICU until the patient was stable enough to tolerate fluid removal. A priori criteria for commencement of PD cycles were agreed upon by all intensivists and included no fluid bolus given within the past 4 hours, no escalation of inotropes or vasopressors within the last 2 hours, VIS $\leq 15$, and oxygen saturations $\geq 65 \%$ on inspired oxygen fraction $\leq 0.4$. PD consisted initially of $10 \mathrm{~mL} / \mathrm{kg}$ dwell volumes with initial $1.5 \%$ dextrose concentration and 60-minute manual cycles (in/dwell 40 minutes, drain 20 minutes). Standard bicarbonate-based (lactate free) dialysate solutions were prepared under sterile conditions in our pharmacy. Dextrose concentration was increased $(2.5 \%, 4.25 \%)$, if necessary, to achieve the desired net negative fluid balance with tolerable hemodynamics. Potassium was added to the dialysate solution at 0 to $4 \mathrm{mmol} / \mathrm{L}$ to target a serum potassium concentration of 3.5 to $4.5 \mathrm{mEq} / \mathrm{L}$. We do not add heparin routinely to dialysate fluid. Dialysate bags and tubing were changed every 72 hours. PD losses were not routinely replaced with volume boluses. Net daily fluid balance was calculated as the difference between the total fluid intake and total fluid output per 24-hour period. Total fluid output was calculated as the sum of urine output, stool losses, PD ultrafiltrate, chest tube output, bloodwork, and nasogastric tube losses. Cycles were later adjusted from 30 to 60 minutes as necessary. Dialysate fluid was sent 3 times a week for cell count, gram stain, and culture. Termination of PD and removal of the PDC occurred when the patient was in a weaning phase defined as a net negative fluid balance for a minimum of 2 consecutive days, decreasing ventilator and inotropic support (VIS $\leq 10)$, normal serum potassium $(<4.0 \mathrm{mEq} / \mathrm{L})$, and urine output $\geq 1 \mathrm{~mL} / \mathrm{kg} / \mathrm{h}$ with PD on hold (with or without diuretic therapy).

Study participants who were randomized to the nonprophylactic PDC group were treated by standard care according to their attending physician. At our institution, diuresis is typically initiated with furosemide, either by continuous infusion or bolus dosing at the discretion of the treating team. Study design allowed for placement of a PDC if clinically indicated due to recalcitrant hyperkalemia (serum potassium $>5.9 \mathrm{mEq} / \mathrm{L}$ ), volume overload unresponsive to diuretics, abdominal compartment syndrome, oliguria (urine output $<1 \mathrm{~mL} / \mathrm{kg} / \mathrm{h}$ ) for more than 4 hours despite medical intervention, or increased serum creatinine in association with persistent metabolic acidosis or low cardiac output syndrome (LCOS).

Oxygen consumption was measured continuously by respiratory mass spectrometry (AMIS2000, Innovision A/S, Odense, Denmark). Patients were intubated with a cuffed endotracheal tube whenever possible and received time-cycled volume ventilation with pressure support. Ventilation volume and rate were controlled to maintain arterial carbon dioxide tension 35 to $45 \mathrm{~mm} \mathrm{Hg}$. Inspired oxygen fraction was adjusted as needed to maintain an arterial saturation of $65 \%$ to $80 \%$. Central temperature was maintained between $36^{\circ} \mathrm{C}$ and $37^{\circ} \mathrm{C}$. Sedation was maintained with continuous, intravenous infusions of morphine or fentanyl as well as intermittent intravenous injections of lorazepam, morphine, or fentanyl. When indicated, rocuronium was used for muscle relaxation. Inotropic and vasoactive agents were administered as continuous infusions during the study, and were adjusted by the bedside clinicians based on the patient's hemodynamic status.

Systemic arterial and venous pressures and oxygen saturations were monitored through arterial and internal jugular venous lines placed in the operating room according to routine practice. There were no additional blood draws for the study protocol. Standard blood gases were performed.

The primary outcome was time until the first postoperative day of negative fluid balance. Secondary outcomes included $\mathrm{Q}_{\mathrm{s}}, \mathrm{SVRi}$, time to lactate $\leq 2 \mathrm{mmol} / \mathrm{L}$, maximum VIS on postoperative days 2 to 5 , time to sternal closure, time to first extubation, modified clinical outcome score, ${ }^{16}$ and hospital LOS.

\section{Adverse Events}

Death, need for CPR or ECLS, stroke, bowel obstruction, bowel perforation, omental herniation, and peritonitis were all considered to be serious adverse events and were tracked. An independent external data safety monitoring board was in place to oversee the safety of the study 
participants. The data safety monitoring board was composed of 5 members (ie, a biostatistician, bioethicist, pediatric cardiac intensivist, pediatric cardiac surgeon, and pediatric cardiologist) who had access to all the data. They were informed of all serious adverse events within 1 work day. There was an interim independent analysis for safety outcomes half way through recruitment.

\section{Statistical Analysis}

We calculated that enrollment of 22 patients would provide statistical power of $80 \%$ to detect a 24 -hour reduction in the time to negative fluid balance (the minimal clinically important difference) assuming a standard deviation of 20 hours or approximately 0.83 days (based on institutional published data for the mean first postoperative day of negative balance of $2.7 \pm 0.83$ days), with an alpha (type 1 error rate) of $0.05 .^{18}$ The primary outcome for our study compared the time to the first postoperative day of negative fluid balance between the groups with and without prophylactic PDC, and was performed using the $t$ test. Continuous data are presented as means with standard deviation or median with interquartile range depending on the normality of the distribution. Normally distributed data were analyzed using the $t$ test and nonnormally distributed data were analyzed using the Wilcoxon rank sum test. Categorical data is presented as counts with percentages and was analyzed using the Fisher exact test. Repeated measures analysis of variance was used to assess the effect of time or group on SVRi. All analyses were based on an intention-to-treat approach. A 2-sided $P$ value of $<.05$ was used for statistical significance. Statistical analysis was performed using SAS version 9.2 (SAS Institute Inc, Cary, NC).

\section{RESULTS}

Enrollment began in September 2010 and ended in July 2013 (Figure 1). Enrollment of patients was not consecutive due to competing studies. No patients were excluded due to prematurity, weight, or urine output criteria. A total of 22 patients underwent randomization: 10 were randomized to prophylactic PDC and 12 to standard care. The baseline characteristics of the 2 groups were similar (Table 1). There were no clinically important differences between groups in regard to weight, cardiac anatomical risk factors (eg, aortic atresia with mitral stenosis, moderate or severe right ventricular dysfunction, pulmonary venous obstruction, intact or restrictive atrial septum, and ascending aorta $<2 \mathrm{~mm}$ ) or cardiopulmonary bypass time. Patients randomized to prophylactic PDC had a higher preoperative mean peak lactate. None of the participants had left or right atrial isomerism, left ventricular coronary fistulae, a dysplastic pulmonary valve with moderate or severe regurgitation, a common atrioventricular valve. or more than mild atrioventricular valve insufficiency. No participants randomized to standard care required crossover to a PDC. The mean time to initiation of PD cycles in the intervention group was $14.6 \pm 6.3$ hours after arrival in the PCICU. Among 4 patients randomized to prophylactic PDC insertion, the catheter was left open to passive drainage because they did not meet criteria for dialysis. The mean drainage by the PDC of that group was $54.9 \pm 13.5 \mathrm{~mL} / \mathrm{kg}$ in the first 24 hours. There was no statistically significant difference in chest drain output or fluid intake between the two groups during the first 48 hours. All but 1 of the participants (21 out of 22) survived to hospital discharge.

The mean time to first postoperative negative fluid balance was $2.70 \pm 1.06$ days for the prophylactic PDC group and $2.67 \pm 0.65$ days for the standard care group $(P=.93)$. Furthermore, prophylactic PDC did not confer any benefit compared to standard care with regard to $Q_{s}$ (Figure 2) or SVRi (Figure 3) at any time point. There was no significant effect of either time $(P=.92)$ or group

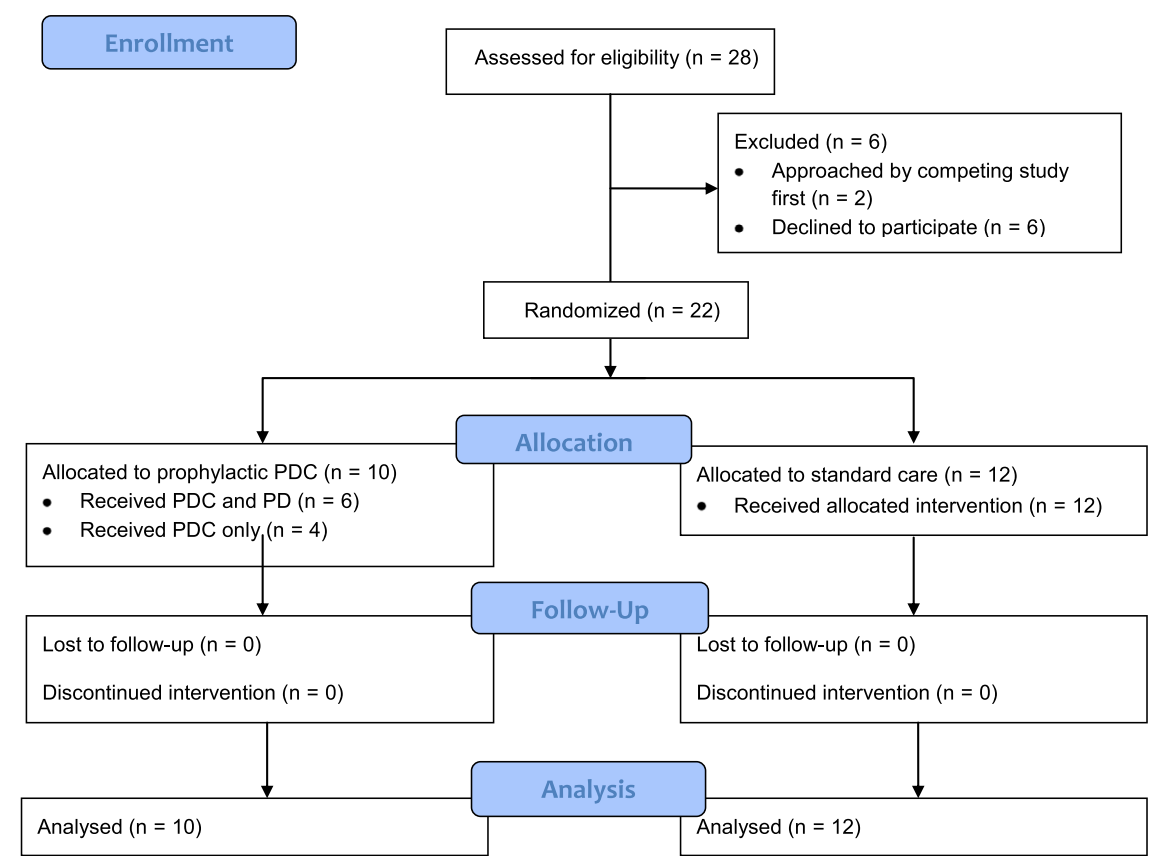

FIGURE 1. CONSORT flow diagram. $P D C$, Peritoneal dialysis catheter. 
TABLE 1. Baseline characteristics

\begin{tabular}{|c|c|c|}
\hline Variable & $\begin{array}{l}\text { Prophylactic } \\
\text { PDC }(\mathbf{n}=10)\end{array}$ & $\begin{array}{c}\text { Standard } \\
\text { care }(n=12)\end{array}$ \\
\hline Weight (kg) & $3.33 \pm 0.60$ & $3.35 \pm 0.46$ \\
\hline Male sex, n (\%) & $5(50)$ & $10(83)$ \\
\hline Preoperative ventilation days & $3.8 \pm 3.5$ & $4.3 \pm 5.8$ \\
\hline Aortic atresia with mitral stenosis & $5(50)$ & $4(33)$ \\
\hline $\begin{array}{l}\text { Moderate or severe right ventricular } \\
\text { dysfunction }\end{array}$ & $3(30)$ & $1(8)$ \\
\hline Pulmonary venous obstruction & $0(0)$ & $1(8)$ \\
\hline Intact or restrictive atrial septum & $1(10)$ & $1(8)$ \\
\hline Ascending aorta $<2 \mathrm{~mm}$ & $2(20)$ & $3(25)$ \\
\hline Peak preoperative lactate & $7.47 \pm 7.66$ & $3.50 \pm 2.67$ \\
\hline Lowest preoperative $\mathrm{pH}$ & $7.22 \pm 0.14$ & $7.31 \pm 0.06^{*}$ \\
\hline Lowest preoperative base deficit & $-3.06 \pm 6.65$ & $-2.35 \pm 5.63^{*}$ \\
\hline $\begin{array}{l}\text { Lowest preoperative arterial oxygen } \\
\text { tension }\end{array}$ & $32.2 \pm 8.0 \dagger$ & $38.9 \pm 5.8 \ddagger$ \\
\hline $\begin{array}{l}\text { Peak preoperative vasoactive inotrope } \\
\text { score }\end{array}$ & $9.3 \pm 9.1$ & $8.7 \pm 5.7$ \\
\hline Age at time of Norwood (d) & $9.2 \pm 4.0$ & $9.4 \pm 5.5$ \\
\hline Cardiopulmonary bypass time (min) & $124 \pm 48$ & $114 \pm 46$ \\
\hline Repeat cardiopulmonary bypass & $4(40)$ & $3(25)$ \\
\hline ACC time (min) & $48.2 \pm 30.1$ & $52.3 \pm 21.4$ \\
\hline Deep hypothermic circulatory arrest & $9(90)$ & $12(100)$ \\
\hline $\begin{array}{l}\text { Deep hypothermic circulatory arrest } \\
\text { time (min) }\end{array}$ & $15.2 \pm 10.0 \dagger$ & $14.3 \pm 11.5$ \\
\hline Modified ultrafiltration time (min) & $8.5 \pm 0.8 \S$ & $6.8 \pm 2.5 \ddagger$ \\
\hline
\end{tabular}

$(P=.79)$ on SVRi. There was also no difference between the 2 groups in terms of time to lactate $\leq 2 \mathrm{mmol} / \mathrm{L}$, maximum VIS on postoperative days 2 to 5 , time to sternal closure, time to first extubation, modified clinical outcome score, or hospital LOS (Table 2).

Four patients randomized to prophylactic PDC had 1 or more serious adverse events; 4 required postoperative CPR and 3 required ECLS. No patient in the control group experienced a serious adverse event. Of 3 patients who required ECLS, 1 was cannulated 4 hours postoperatively for LCOS believed to be secondary to neoaortic valve insufficiency. He was randomized to prophylactic PDC, but had not been dialyzed. PD was started on ECLS and was then

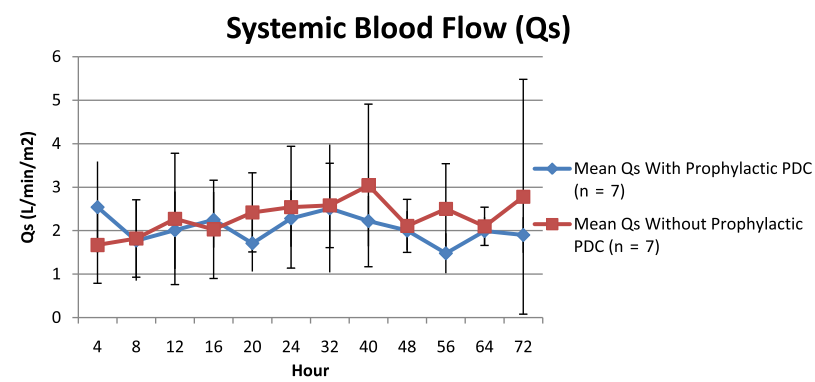

FIGURE 2. Mean indexed systemic blood flow $\left(Q_{s}\right)$. Error bars indicate 1 standard deviation. $P D C$, Peritoneal dialysis catheter.
Indexed Systemic Vascular Resistance (SVRi)

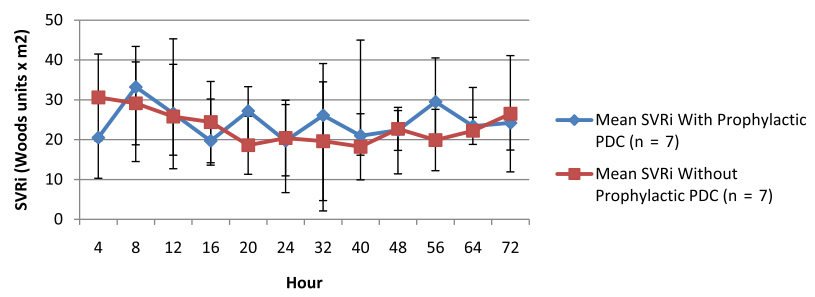

FIGURE 3. Mean indexed systemic vascular resistance (SVRi). Error bars indicate 1 standard deviation. $P D C$, Peritoneal dialysis catheter.

converted to continuous renal replacement therapy. $\mathrm{He}$ was diagnosed with a chromosomal abnormality (trisomy 15 and partial chromosome 6 deletion) postoperatively and was not listed for heart transplantation due to the combination of midline defects and poor neurodevelopmental prognosis. He was decannulated after a 14-day ECLS run, but died 3 days later of progressive LCOS. The second patient required extracorporeal CPR on her first postoperative night after a sudden arrest associated with increasing blood lactate levels and bleeding. PD cycles had started and cardiac arrest occurred during the second dwell. The preoperative course had been complicated by supraventricular tachycardia and treated with propranolol. There was no supraventricular tachycardia recurrence postoperatively, before the arrest. The patient survived to ECLS decannulation and hospital discharge. The third patient had a sudden cardiac arrest requiring extracorporeal CPR the first postoperative night. She was randomized to prophylactic PDC, but had not been dialyzed. ECLS was complicated by a cerebral stroke. The patient survived to ECLS decannulation and hospital discharge and has since had a bidirectional Glenn procedure and then orthotopic heart transplant for decreased ventricular function. The fourth patient developed cardiac tamponade within hours after Norwood requiring CPR, mediastinal exploration, and thrombus evacuation. He did not require ECLS. No participants had peritonitis, bowel obstruction, bowel perforation, or omental herniation secondary to the PDC. Minor complications of PDC were hyperglycemia $(\mathrm{n}=2)$, hypokalemia $(n=2)$, catheter leakage $(n=2)$, and transient retention of PD fluid $(n=4)$.

We performed a post-hoc secondary analysis with the ECLS patients excluded because ECLS may alter regulation and management of fluid balance. The mean time to first postoperative negative fluid balance was $2.29 \pm 0.95$ days for the prophylactic PDC group $(\mathrm{n}=7)$ and $2.67 \pm 0.65$ days for the standard care group $(\mathrm{n}=12)(P=.31)$.

\section{DISCUSSION}

To our knowledge, ours is the first randomized controlled trial of prophylactic PDC after Norwood procedure in patients with HLHS. Prophylactic PDC, with or without 
TABLE 2. Study outcomes and serious adverse events, according to study group

\begin{tabular}{|c|c|c|c|}
\hline Variable & Prophylactic PDC $(\mathbf{n}=10)$ & Standard care $(n=12)$ & $P$ valu \\
\hline \multicolumn{4}{|l|}{ Primary outcome } \\
\hline Time to first postoperative negative fluid balance (d) & $2.70 \pm 1.06$ & $2.67 \pm 0.65$ & .93 \\
\hline \multicolumn{4}{|l|}{ Secondary outcome } \\
\hline Time to lactate $\leq 2 \mathrm{mmol} / \mathrm{L}(\mathrm{h})$ & $15.5 \pm 10.1$ & $20.3 \pm 8.3$ & .23 \\
\hline Maximum vasoactive-inotrope score on postoperative day $2-5$ & $16.4 \pm 9.8$ & $15.0 \pm 5.3$ & 67 \\
\hline Time to sternal closure (d) & $5.33 \pm 2.87^{*}$ & $4.17 \pm 1.12$ & .21 \\
\hline Time to first extubation (d) & $10.1 \pm 4.1 *$ & $8.6 \pm 4.9$ & .46 \\
\hline Hospital length of stay (d) & $20.4 \pm 10.8$ & $27.7 \pm 21.4$ & .34 \\
\hline Modified clinical outcome score $\dagger$ & $4.70 \pm 2.00$ & $4.25 \pm 1.36$ & .54 \\
\hline Patients with serious adverse event $\ddagger$ & $4(40)$ & 0 & .03 \\
\hline
\end{tabular}

Values are presented as mean \pm standard deviation or $\mathrm{n}(\%) . P D C$, Peritoneal dialysis catheter. $* \mathrm{n}=9$. $\dagger$ Range, $0-7$. $\ddagger$ Four cardiac arrests, 3 of which required extracorporeal life support, and 1 of which resulted in death.

dialysis (using a priori criteria), did not decrease the time to a negative fluid balance as compared with standard care, our study's primary outcome. PDC insertion and peritoneal drainage, without PD cycles, signified an intervention as this group had mean drainage of $54.9 \mathrm{~mL} / \mathrm{kg}$ in the first 24 hours. No benefit was demonstrated with any of the secondary outcomes.

In contrast to other studies, our study did not find any benefit from prophylactic PDC on fluid balance and is the second study to indicate potential harm from PDC. A retrospective cohort study of 146 neonates and infants found that early PD (within 24 hours of cardiac surgery) was associated with a significant decrease in mortality compared with delayed $\mathrm{PD}^{19}$; however, there was also a high incidence of severe complications $(9.6 \%)$ secondary to PD. These results must be interpreted in the context of 30 - and 90-day mortality of $28.1 \%$ and $36.3 \%$, respectively. Another retrospective study of 36 infants after complete atrioventricular septal defect repair reported a decreased time to reach negative fluid balance in the group receiving passive peritoneal drainage. ${ }^{20}$ Complications secondary to the PDC were not noted. Neither of these 2 studies included any patients with HLHS and the difference in results may be attributed to differences in postoperative physiology. We suggest that unlike patients who have undergone biventricular repairs, patients who have undergone a modified Norwood are less tolerant of fluctuations in volume status, being hemodynamically very sensitive to both decreased or fluctuating volume status and early fluid removal. The differences may be related to differences in the surgical procedure, especially the absence of right ventricular restrictive physiology that accompanies many neonatal biventricular repairs. The differences between our cohort and those reported above are that the Norwood operation does not involve ventricular septal defect closure, the right ventricle to pulmonary artery shunt is pressure restrictive and the right ventricular volume load from pulmonary insufficiency is much less. Indeed the adverse factors after the Norwood are due to increased SVRi and increased ineffective pulmonary blood flow both of which are poorly tolerated in the early postoperative period. ${ }^{21,22}$ In addition, all of our patients were neonates and age-related differences may be important.

Averbuch and colleagues ${ }^{23}$ retrospectively reported on 17 neonates after cardiopulmonary bypass and congenital heart surgery who received percutaneous PDC insertion and drainage for deteriorating ventilator function or significant oliguria. Their series included 5 neonates after Norwood procedure. Timing of catheter insertion was not reported. Ventilation and oxygenation were improved compared with precatheter insertion. One patient developed necrotizing enterocolitis; mortality was not reported. Sasser and colleagues $^{24}$ described the use of prophylactic PD compared with passive peritoneal drainage and diuretics in high-risk neonates and infants after cardiopulmonary bypass in a prospective nonrandomized cohort study. They found that the use of prophylactic PD was associated with a decreased incidence of severe fluid overload in the early postoperative period. Sternal closure occurred earlier in the prophylactic PD group, but PCICU LOS was not different between groups. This study had a smaller proportion of Norwood patients in the prophylactic PD group (24\%) compared with the control group (48\%), making comparison difficult. Similar to our results, they describe a low incidence of severe complications (peritonitis and omental herniation, 1 each), but there were 4 deaths in the study, including 3 in the prophylactic PD group (3 out of $25 ; 12 \%$ ) thought unlikely to be directly influenced by the use of PD.

In our study there was no difference in the time to a negative fluid balance between the 2 groups. Previous data from our institution described the first postoperative day of negative fluid balance was 2.7 days after modified Norwood procedure. ${ }^{18}$ Insertion of a PDC has not altered this timing. There was no difference in $\mathrm{Q}_{\mathrm{s}}$ or SVRi between the 2 groups, suggesting that either our population did not have increased intra-abdominal pressures or that the use of passive peritoneal drainage or prophylactic PD does not alter preload or afterload to single ventricle physiology. There was no difference in the peak VIS between 
groups, suggesting that prophylactic PDC did not improve tenuous hemodynamics in the early postoperative period.

The incidence of major complications due to PDC such as small bowel obstruction, peritonitis, wound dehiscence, omental herniation, and bowel perforation ranges from $5 \%$ to $7 \%{ }^{2-5}$ and is less $(4.3 \%-4.8 \%)$ in those patients in whom the PD catheter was inserted intraoperatively. 8,14 None of our patients had any of these severe complications. We included death, need for CPR or ECLS, and stroke as serious adverse events. In the patient who had a cardiac arrest during a period of PD dwell, it is possible that the arrest was secondary to impairment of preload.

The strengths of this study include a homogenous, highrisk population and a prospective, randomized design. The use of continuous mass spectrometry to evaluate $Q_{s}$ and SVRi provides additional objective and relevant information about this fragile population. A limitation of this study is its single center design. A second limitation is that the intervention could not be blinded. In addition, management of the patients was not protocolized. These 2 factors could have introduced performance bias. Nevertheless, the results reflect the efficacy of PDC in real-life postoperative settings where care is at the discretion of the treating medical team. All participants appropriately received the intervention; in 4 patients, the PDC was left open to drain and PD never started. Criteria for initiation of PD were based on consensus of expert opinion at our center given that there are no published criteria on starting PD. Due to a priori criteria for initiation of PD, dialysis cycles may not have been started early enough to alter fluid balance. This study was not powered to detect a shorter reduction of time to negative fluid balance because we suggest that a 24-hour reduction is the minimum clinically important difference to warrant the potential morbidity of PD catheter placement. Patients in the PDC group may have been sicker, which could account for the adverse events and lack of benefit found; they had a higher preoperative peak lactate and there was a higher incidence of preoperative right ventricular dysfunction. We only have $Q_{s}$ and SVRi data on 14 patients (7 in each group) due to the presence of ECLS in 3 patients and missing data points (eg, hemoglobin, venous oxygen saturation, or oxygen consumption) in the remaining 5 patients that prevented complete calculations.

\section{CONCLUSIONS}

The strategy of prophylactic PDC with or without dialysis (based on a priori criteria) did not decrease the time to achieve a negative fluid balance after the Norwood procedure, did not alter physiologic variables postoperatively and was associated with more severe adverse events. The differences in our study compared with previous reports are most likely related to the age of our patients and important differences in postoperative physiology. Early attempts to achieve a negative fluid balance using PDC after the Norwood procedure may be associated with an increased risk of adverse outcomes.

The authors thank the members of the external data safety monitoring board for their time and thoughtful review (Dr Thomas Kulik, chair, Children's Hospital Boston; Dr David Patton, Alberta Children's Hospital; Dr Jennifer Hirsch, University of Michigan; Dr Iain Mitchell, Alberta Children's Hospital; and Dr James Gurney, University of Memphis). The authors also thank Dr Long Guo and Dr Yong Cui for setting up and running the mass spectrometer for our study participants.

\section{References}

1. Norwood WI, Kirklin JK, Sanders SP. Hypoplastic left heart syndrome: experience with palliative surgery. Am J Cardiol. 1980;45:87-91.

2. Werner HA, Wensley DF, Lirenman DS, LeBlanc JG. Peritoneal dialysis in children after cardiopulmonary bypass. J Thorac Cardiovasc Surg. 1997;113: 64-8.

3. Chan KL, Ip P, Chiu CS, Cheung YF. Peritoneal dialysis after surgery for congenital heart disease in infants and young children. Ann Thorac Surg. 2003;76: 1443-9.

4. Dittrich S, Dahnert I, Vogel M, Stiller B, Haas NA, Alexi-Meskishvili V, et al. Peritoneal dialysis after infant open heart surgery: observations in 27 patients. Ann Thorac Surg. 1999;68:160-3.

5. Pedersen KR, Hjortdal VE, Christensen S, Pedersen J, Hjortholm K, Larsen SH, et al. Clinical outcome in children with acute renal failure treated with peritoneal dialysis after surgery for congenital heart disease. Kidney Intl. 2008;73:S81-6.

6. Alkan T, Akcevin A, Turkoglu H, Paker T, Sasmazel A, Bayer V, et al. Postoperative peritoneal dialysis in neonates and infants after complex congenital cardiac surgery. ASAIO J. 2006;52:693-7.

7. Boigner H, Brannath W, Hermon M, Stoll E, Burda G, Trittenwein G, et al. Predictors of mortality at initiation of peritoneal dialysis in children after cardiac surgery. Ann Thorac Surg. 2004;77:61-5.

8. Sorof JM, Stromberg D, Brewer ED, Feltes TF, Fraser CD. Early initiation of peritoneal dialysis after surgical repair of congenital heart disease. Pediatr Nephrol. 1999;13:641-5.

9. Giuffre RM, Tam KH, Williams WW, Freedom RM. Acute renal failure complicating pediatric cardiac surgery: a comparison of survivors and nonsurvivors following acute peritoneal dialysis. Pediatr Cardiol. 1992;13:208-13.

10. Verrier ED, Boyle EM. Endothelial cell injury in cardiovascular surgery. Ann Thorac Surg. 1996;62:915-22.

11. Olofsson PH, Berg S, Ahn HC, Brudin LH, Vikstrom T, Johansson KJ. Gastrointestinal microcirculation and cardiopulmonary function during experimentally increased intra-abdominal pressure. Crit Care Med. 2009;37:230-9.

12. Wauters J, Claus P, Brosens N, McLaughlin M, Malbrain M, Wilmer A. Pathophysiology of renal hemodynamics and renal cortical microcirculation in a porcine model of elevated intra-abdominal pressure. J Trauma. 2009;66:713-9.

13. Hazle MA, Gajarski RG, Yu S, Donohue J, Blatt NB. Fluid overload in infants following congenital heart surgery. Pediatr Crit Care Med. 2013;14:44-9.

14. Stromberg D, Fraser CD, Sorof JM, Drescher K, Feltes TF. Peritoneal dialysis: an adjunct to pediatric postcardiotomy fluid management. Tex Heart Inst J. 1997;24: 269-77.

15. Gaies MG, Gurney JG, Yen AH, Napoli ML, Gajarski RJ, Ohye RG, et al Vasoactive-inotrope score as a predictor of morbidity and mortality in infants after cardiopulmonary bypass. Pediatr Crit Care Med. 2010;11:234-8.

16. Mackie AS, Alton GY, Dinu IA, Joffe AR, Roth SJ, Newburger JW, et al. Clinical outcome score predicts the need for neurodevelopmental intervention after infant heart surgery. J Thorac Cardiovasc Surg. 2013;145:1248-54.

17. Murala JS, Singappuli K, Provenzano SC, Nunn G. Techniques of inserting peritoneal dialysis catheters in neonates and infants undergoing open heart surgery. J Thorac Cardiovasc Surg. 2010;139:503-5.

18. Atallah J, Dinu IA, Joffe AR, Robertson CM, Sauve RS, Dyck JD, et al. Two-year survival and mental and psychomotor outcomes after the Norwood procedure: an analysis of the modified Blalock-Taussig shunt and right ventricle-to-pulmonary artery shunt surgical eras. Circulation. 2008;118:1410-8. 
19. Saini A, Delius RE, Sheshadri S, Walters H, Mastropietro CW. Passive peritoneal drainage improves fluid balance after surgery for congenital heart disease. Eur J Cardiothorac Surg. 2012;41:256-60.

20. Bojan M, Gioanni S, Vouhe PR, Journois D, Pouard P. Early initiation of peritoneal dialysis in neonates and infants with acute kidney injury following cardiac surgery is associated with a significant decrease in mortality. Kidney Int. 2012;82: 474-81.

21. Tweddell JS, Hoffman GM, Fedderly RT, Berger S, Thomas JP, Ghanayem NS, et al. Phenoxybenzamine improves systemic oxygen delivery after the Norwood procedure. Ann Thorac Surg. 1999;6:161-7.
22. Tweddell JS, Hoffman GM, Mussatto KA, Fedderly RT, Berger S, Jaquiss RD, et al Improved survival of patients undergoing palliation of hypoplastic left heart syndrome: lessons learned from 115 consecutive patients. Circulation. 2002; 106:I82-9. 23. Averbuch N, Birk E, Frenkel G, Gogia O, Shulman OR, Bruckheimer E, et al Percutaenous intraperitoneal catheters in neonates following open heart surgery. J Intensive Care Med. 2014:29:160-4.

24. Sasser WC, Dabal RJ, Askenazi DJ, Borasino S, Moellinger AB, Kirklin JK, et al Prophylactic peritoneal dialysis following cardiopulmonary bypass is children is associated with decreased inflammation and improved clinical outcomes. Conge nit Heart Dis. 2014;9:106-15.

\title{
EDITORIAL COMMENTARY
}

\section{Prophylactic peritoneal dialysis after the Norwood procedure: Worth the risk?}

\author{
Jonathan M. Chen, MD
}

See related article on pages 222-8.

Despite iterative, incremental improvements in the mortality rates associated with complex newborn cardiac surgery, persistent morbidities related to perioperative care continue to plague intensive care unit and hospital lengths of stay, not to mention influence overall survival for these patients. Increasingly, the willingness of congenital heart surgeons to perform such procedures for neonates with low birthweight, those who are premature, and those with other syndromes has further magnified some of these most basic obstacles, few as fundamental as the challenge of establishing early perioperative negative fluid balance without jeopardizing cardiac output. In this issue of the Journal, Ryerson and colleagues from Stollery Children's Hospital ${ }^{1}$ describe the first randomized controlled trial of prophylactic peritoneal dialysis (PD) after the Norwood procedure. Previous retrospective studies evaluating the use of PD catheters for recipients of complex newborn repairs have examined varying aspects of their implementation (passive drainage vs dialysis, early vs late insertion, and presence vs absence

From Seattle Children's Hospital/University of Washington School of Medicine, Seattle, Wash.

Disclosures: Author has nothing to disclose with regard to commercial support.

Received for publication Sept 3, 2014; accepted for publication Sept 4, 2014; available ahead of print Oct 1, 2014.

Address for reprints: Jonathan M. Chen, MD, Seattle Children's Hospital/University of Washington School of Medicine, 4800 Sand Point Way, NE, Seattle, WA 98105 (E-mail: jmchen@uw.edu).

J Thorac Cardiovasc Surg 2015;149:228-9

$0022-5223 / \$ 36.00$

Copyright (c) 2015 by The American Association for Thoracic Surgery

http://dx.doi.org/10.1016/j.jtcvs.2014.09.002 of acute kidney injury), and although their results have largely supported prior beliefs regarding the use of PD catheters in perioperative settings, none has been studied in such a finite cohort of arguably some of the most hemodynamically fragile neonatal cardiac patients. ${ }^{2,3}$

As Ryerson and colleagues ${ }^{1}$ concede, it was not possible for this to be a blinded study, and in that sense, because the management of the patients was not protocolized (although commencement of PD was overseen by a priori criteria), there is always a possibility of performance bias. Nevertheless, the lack of statistical improvement of either primary (negative fluid balance) or secondary end points-in concert with a striking rate of adverse events-certainly bring to mind the concept of no free lunch. The fact that this study cohort is somewhat different from those historically thought to benefit most from perioperative PD (ie, newborn tetralogy repairs and patients with restrictive right ventricular physiology) is slightly tempered by the Stollery group's universal use of right ventricle and pulmonary artery conduits in their cohort, where the likelihood of early aggressive fluid resuscitation (potentially in combination with right ventricular diastolic dysfunction) was no doubt considerable. Although negative fluid balance as a primary end point may seem less clinically powerful than more traditional outcome variables, it does represent an objective target, with fewer confounding influences.

Accordingly, Ryerson and colleagues ${ }^{1}$ chose to include all-cause morbidities in the study group, and in doing so report not just the more peritoneal morbidities commonly associated with PD catheters, as was done by Sasser and colleagues, ${ }^{2}$ but also the major adverse events that themselves either could have been related to the PD infusion (with resultant effect on both preload and pulmonary vascular resistance), or could have negatively biased the 